\title{
Percepciones, Actitudes y Prácticas en Malaria en el Amazonas Colombiano
}

\author{
Francy Pineda G ${ }^{\mathbf{1}}$. y Carlos A. Agudelo ${ }^{2}$ \\ ${ }^{1}$ Bacterióloga. M. Sc. Infecciones y Salud en el Trópico. Instituto de Salud Pública, facultad \\ de Medicina, Universidad Nacional de Colombia. E-mail: fypinedag@unal.edu.co \\ ${ }^{2}$ Médico. M.Sc. Salud Pública, M. Sc. Ciencias. Instituto de Salud Pública. Facultad de \\ Medicina. Universidad Nacional de Colombia. E-mail: caagudeloc@unal.edu.co
}

Recibido 18 Febrero 2004/Enviado para Modificación 3 Diciembre 2004/Aceptado 22Julio 2005

\section{RESUMEN}

Objetivo El objetivo del estudio fue obtener información sobre las percepciones, actitudes y las prácticas que las personas del Amazonas Colombiano tienen sobre la etiología de la malaria, el diagnóstico, la profilaxis, la terapéutica, la prevención, y la percepción del riesgo para contraer la malaria.

Métodos Se realizó un estudio cualitativo y se utilizó como técnica de investigación grupos focales, en total se realizaron 23 , cada uno contó con la participación de 6 a 10 personas clasificadas de acuerdo a unas variables de inclusión pertinentes para el estudio.

Resultados El estudio encontró que las personas que mejor conocen las medidas preventivas y de control son quienes tienen un riesgo alto para adquirir malaria, pero sin embargo no las ponen en práctica. Existen dificultades de acceso al diagnóstico y tratamiento de la malaria y problemas de automedicación en poblaciones de alto riesgo.

Conclusiones Los factores comportamentales de las poblaciones expuesta a la malaria, pueden facilitar u obstaculizar las intervenciones de control en el Departamento del Amazonas Colombiano.

Palabras Clave: Malaria, actitudes, conocimieto (fuente:DeCS, BIREME).

\section{ABSTRACT}

Perceptions, practice and attitudes concerning malaria in the Colombian Amazon region

Objective Obtaining information about the perceptions, attitudes and practices which people living in the Colombian Amazon region hold about the aetiology of malaria, its diagnosis, prophylaxis, therapy, prevention and their perception concerning the risk of contracting malaria. 
Methods A qualitative study was carried out; focal groups were used as the research technique. A total of 23 were held, each one involving 6 to 10 people classified according to some inclusion variables which were pertinent for the study.

Results The study revealed that the people having the best knowledge of preventative and control measures were those having a high risk of acquiring malaria; however, they did not put them into practice. There are difficulties in gaining access to diagnosis and treatment of malaria and problems of self-medication in high risk populations.

Conclusions The behavioural factors of those populations exposed to malaria could facilitate and/or hamper control interventions in Colombia's Amazonas Department.

Key Words: Malaria, perception, attitude, practice (source: MeSH,NLM).

L

os programas de control de la malaria, implementados antes de la década de los años 90, no tuvieron en cuenta el conocimiento de aspectos comportamentales y socioculturales de las poblaciones afectadas por la malaria. Con el resurgimiento de la malaria, a partir de esta década, la OMS incluyó el estudio de estos para controlar la malaria con su iniciativa Roll Back Malaria(RBM) (1).

El conocimiento que las personas tienen de la malaria es el producto de lo aprendido en su experiencia individual, grupal y social. Este aprendizaje ocurre dentro de una comunidad y un marco cultural o contexto de vida que brindan las pautas para que las personas actúen o tengan una percepción frente a la malaria $(2,3)$.

La modificación de la transmisión de la malaria y su control puede tener buenos resultados con modelos basados en el conocimiento de las percepciones, las actitudes y las prácticas, y de esta manera se puede lograr el desarrollo de programas de control sostenible y adaptado a las necesidades locales.

Este estudio es exploratorio y pretende describir las percepciones, las actitudes y las prácticas que las personas del Departamento del Amazonas Colombiano tienen sobre la malaria.

\section{MÉTODOS}

El estudio se realizó en el Departamento del Amazonas Colombiano, una región que se encuentra debajo de los 1500 metros sobre el nivel del mar con 
temperatura media de $29^{\circ} \mathrm{C}$ y promedio de lluvias anuales de 3000 $\mathrm{mm}$ /año, condiciones propicias para la transmisión de la malaria.

Para este estudio incluyeron poblaciones en zonas de alto riesgo para la transmisión de malaria como Tarapacá y La Pedrera junto con poblaciones en zonas de mediano y bajo riesgo como los municipios de Leticia y Puerto Nariño. En estas zonas un porcentaje importante de la población es indígena; predominan las etnias Tikuna, Uitoto, Yucunas, Tanimuka y Mirañas que están ubicadas en los resguardos indígenas del trapecio amazónico, Puerto Nariño, Comeyafú y Tarapacá (4).

Se realizó un estudio cualitativo y se utilizó como técnica principal de investigación los grupos focales que son grupos de discusión en donde emergen diferencias, consensos y significaciones entre los participantes (5).

La discusión con los grupos se estructuró en torno a tópicos claves definidos previamente. Estos tópicos fueron: Nombres con que reconocen la enfermedad, síntomas que se asocian, tratamientos que utilizan, diagnóstico, población que reconocen de riesgo, lugares y períodos del día o año que reconocen como períodos de mayor incidencia, medidas preventivas y limitaciones para utilizarlas.

Los grupos focales fueron dirigidos por un investigador, el cual participó únicamente para dar la palabra y como observador. Estas reuniones fueron grabadas para lo cual se informó y solicitó el consentimiento a los participantes. Se organizaron 23 grupos focales, buscando representar las relaciones sociales pertinentes para el estudio, cada uno contó con la participación de 6 a 10 personas. A continuación se indican las variables o atributos que definieron los participantes de cada grupo:

Área: rural y urbana

Sexo: hombres y mujeres

Escolarización: Sin escolarización y con escolarización.

Exposición al riesgo: aserradores y amas de casa

El proceso de análisis de los datos fue descriptivo y clasificatorio a partir de la trascripción de los grupos focales y de las anotaciones tomadas por el investigador. Los criterios de selección de testimonios fueron: saturación de los contenidos, convergencias y divergencias, opiniones aisladas y opiniones mayoritarias. 
A continuación se presenta la información obtenida del análisis de los grupos focales.

Percepciones, actitudes y prácticas relacionadas con la Etiología de la Malaria

Nombres con que reconocen la malaria: No reconocen el término malaria sino paludismo. En Leticia y Puerto Nariño no diferencian entre malaria, dengue y fiebre amarilla.

Causas que enunciaron como origen de la malaria

- Los zancudos (no reconocen el nombre de Anopheles, algunas personas llaman al zancudo "dengue" sin distinguirlo de otros zancudos),

- La falta de aseo y las basuras, están relacionadas con la proliferación de los zancudos.

- La desnutrición (especialmente madres comunitarias y estudiantes de colegio)

- Beber agua contaminada de los ríos

Aunque en La Pedrera reconocen que la transmisión de la malaria se debe a un zancudo, aún conservan mitos de sus antepasados según los cuales la malaria era llamada enfermedad de la chicharra porque esparcía su saliva por la selva, contaminando los alimentos y el agua, pasando cada año por el sector de la Pedrera. El siguiente fragmento muestra parte de un relato de un habitante de la Pedrera:

"Le cae a la comida, al agua, entonces se come eso y ya se infecta un niño, con fiebre, ese tiempo ya marca. Este tiempo ya marca paludismo..."

Comunidad de Comeyafu - Yucuna.

Síntomas que la comunidad asocia con la malaria

Los principales síntomas que asocian a la malaria son la fiebre, el dolor de cabeza, escalofrío, vómito, anemia, sueño, pérdida de apetito y dolor de cabeza.

Los grupos de alto riesgo como los aserradores y las comunidades de Tarapacá y la Pedrera reconocen correctamente los síntomas; incluso creen saber cuando es paludismo causado por $P$. falciparum y $P$. vivax, asociando el primero a síntomas más fuertes.

Población que reconocen como de alto riesgo para contraer la enfermedad 
Los habitantes de las áreas urbanas consideran que ellos no tienen riesgo para adquirir malaria mientras que identifican a las comunidades indígenas, aserradores y pescadores de las zonas rurales como poblaciones de alto riesgo.

Los aserradores y las comunidades indígenas rurales se identifican a sí mismos como población de alto riesgo, consideran que toda la población que habitan sus comunidades está expuesta, y que conviven con esta enfermedad que puede llegar a ser mortal desde hace muchos años.

Esto lo tiene claro un habitante de La Pedrera:

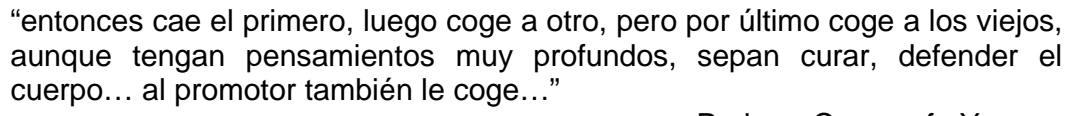

Pedrera Comeyafu Yucuna

Lugares en donde se está más expuesto a contraer la enfermedad

Los grupos estudiados concuerdan que los lugares en donde están más expuestos son las áreas rurales o selváticas, o donde existen estancamientos de agua.

Períodos del día o del año que reconocen como de mayor incidencia

Para las poblaciones estudiadas esta enfermedad no se produce todo el año si no solamente ocurre en algunas épocas, especialmente asociadas a verano que corresponde a los meses de julio, agosto y septiembre.

Percepciones, actitudes y prácticas relacionadas con la prevención de la malaria

Medidas preventivas que conocen

Las relacionadas con la eliminación de los criaderos para los zancudos: el aseo, recogiendo latas, limpiando canales y tanques, evitando tener aguas estancadas, eliminando pozos o charcos. También conocen otras medidas como utilizar toldillo y las fumigaciones.

Las comunidades indígenas refieren que "los pensadores" usaban el calendario ecológico para saber en que época iba a llegar la malaria y realizaban rituales de prevención, además educaban a las personas de la comunidad 
para que no consumieran ciertos alimentos. Debido a que ya no xiste estas prácticas piensan que las epidemias se han hecho más fuertes. Así lo manifiesta este hombre:

“...anteriormente lo que se manejaba era la prevención, anualmente hacíamos curaciones con hoja de tabaco, pinturas, hierbabuena preparada con manicuera o con jugo de piña, es dulce se da especialmente a los niños...

Cacique Puerto Remanso

La única comunidad indígena estudiada que no conocía ningún tipo de medidas preventivas fue la comunidad de Pupuña. Manifestaron que han ido a fumigar pero que no saben para que lo hacen, aunque utilizan medidas tradicionales para espantar los zancudos, como quemar plantas.

Rechazo o limitaciones que tienen para utilizar las medidas preventivas

Las personas no manifiestan rechazo hacia las medidas preventivas; sin embargo, manifiestan que no les agrada que fumiguen sus casas por que, a diferencia del DDT, el insecticida que utilizan ahora les causa alergia.

Es importante resaltar que aunque conocen las medidas preventivas reconocen que muy pocos las ponen en práctica por falta de tiempo, falta de organización del trabajo comunitario y por pereza.

\section{Fumigación}

La población de las comunidades indígenas del área rural de Tarapacá, La Pedrera, Puerto Nariño y Leticia opina que el insecticida que utilizan ahora no es bueno porque no elimina a los zancudos, y por el contrario hace que proliferen más; además perciben que el efecto del DDT era mayor porque mataba gallinas y gatos.

Charlas educativas

Piensan que las charlas que reciben sobre malaria son provechosas pero superficiales, y que las metodologías que utilizan los promotores de salud son aburridas.

Recurso humano que participa en el programa de ETV

A las personas que habitan las comunidades Indígenas de las zonas rurales de Tarapacá y La Pedrera les parece que el recurso humano para realizar la 
fumigación es insuficiente, desmotivado y poco capacitado; manifiestan que no reciben suficiente explicación sobre los trabajos que realizan los técnicos cuando fumigan sus casas. Esto lo resume un habitante de Tarapacá:

"Casi no dicen nada cuando vienen a fumigar, solamente dicen alisten todo, no nos explican mucho del paludismo ni para que sirve la fumigación, pero nosotros ya sabemos para que es, para que maten cucarachas...

Hombre de la comunidad de Caña Brava. Tarapacá

Frecuencia y sostenibilidad de las medidas preventivas

En Puerto Nariño las personas consideran que en la actualidad las medidas preventivas como capacitaciones, fumigaciones y diagnóstico y tratamiento son sostenibles y se realizan frecuentemente. En Tarapacá están satisfechos con la frecuencia de las fumigaciones pero piensan que los controles a los pacientes positivos deberían ser más estrictos.

En La Pedrera y en Leticia consideran que las medidas preventivas como las fumigaciones no son sostenibles y no tienen la frecuencia adecuada; además que el tiempo en el que fumigan no corresponde a sus necesidades. En forma global, opinan que las capacitaciones, fumigaciones y trabajos comunitarios deberían ser más frecuentes y que se deberían hacer para prevenir y no cuando se presente el brote.

Percepciones, actitudes y prácticas relacionadas con el diagnóstico de la Malaria

Accesibilidad a centros de Diagnóstico y tratamiento

Los habitantes del área urbana de Leticia, Tarapacá y Puerto Nariño recurren al hospital de forma rutinaria mientras que las comunidades Indígenas Rurales solamente lo hacen cuando se sienten muy enfermas.

Para las comunidades mas alejadas al área urbana existen problemas de acceso al centro médico y existen limitaciones económicas para que el promotor de salud se desplace al hospital y la escasez de gasolina limita la remisión de las muestras.

Para el grupo de alto riesgo como los aserradores existen problemas como la automedicación y el pago por el tratamiento a sus patronos.

Tratamientos utilizados 
Las comunidades de La Pedrera y Tarapacá identifican los medicamentos y el esquema terapéutico para combatir el paludismo, mientras que en Leticia no.

En cuanto a la efectividad percibida, las comunidades de La Pedrera y de Tarapacá, afirman que el tratamiento no tiene el mismo efecto que antes. Así lo manifiesta un hombre de la Pedrera: "Se presentan mucho caso que han tomado la gota gruesa por primera vez y
le han dado tratamiento y eso no hace caso, vuelve y les brota como a la se-
mana, hasta 8 veces le dan tratamiento y no... Toca que hospitalizarlos y
cambiarles de tratamiento, así se curan..."
Hombre de la comunidad de Angostura. La Pedrera

Otros tratamientos utilizados

Las comunidades rurales estudiadas y los aserradores recurren a los vegetales antes de asistir al hospital, utilizan cáscaras de los árboles de cedro, de costillo, de matarratón o la raíz de azahí; las mujeres utilizan el paico, la albahaca, la sábila o el limón con sal.

En Tarapacá previenen la recaída de la malaria haciendo una dieta especial libre de grasas un mes después de padecerla.

Percepciones, actitudes y prácticas relacionadas con la participación de la comunidad en el programa de control de la malaria

No hay suficiente participación activa de la comunidad en el programa de control de ETV. Los promotores de salud manifiestan que hacer charlas, capacitaciones y trabajos comunitarios no es efectivo porque la comunidad no participa; por su parte, la población manifiesta que les parece importante aprender pero están ocupados para asistir a las reuniones o les causa pereza participar en las jornadas de limpieza que se organizan.

\section{DISCUSIÓN}

El principal hallazgo de este estudio es que la población de la Amazonía Colombiana entrevistada conoce las medidas preventivas y de control para la malaria, especialmente las que tienen que ver con la eliminación de criaderos de zancudos, pero no las ponen en práctica por falta de tiempo, interés y organización comunitaria. 
El estudio coincide con otros estudios realizados en Guatemala y Guyana, los cuales mostraron que la población en riesgo conocía la etiología, los síntomas y el tratamiento (6-7). Sin embargo, existe diferencia tanto con un estudio realizado en 1992 en Colombia donde se estableció que el origen y transmisión de la malaria eran poco conocidos y otro realizado en México que reportó un pobre conocimiento sobre paludismo (8-9).

Otro resultado, es que el acceso a los centros diagnósticos es limitado en las áreas rurales alejadas de las cabeceras municipales, básicamente por problemas económicos que limitan el transporte. McCombie reportó que muchos estudios han establecido que la distancia a los servicios de salud y el costo son las principales razones para la tardanza en el tratamiento de la malaria (10).

También se encontró que la automedicación y la falta de adherencia son lo principales inconvenientes en cuanto al tratamiento, aspecto reportado ampliamente en zonas endémicas para malaria; Forter estima que más de la mitad de las drogas antimaláricas se automedican (11).

Por otro lado, aunque existe conocimiento de las drogas antimaláricas, la población recurre a medidas tradicionales, como infusiones preparadas con corteza de árboles, antes de recurrir al hospital; esta práctica también es reportada en otros estudios (12) en Guatemala el 80\% de la población utiliza medicinas tradicionales de árboles, especialmente bebidas amargas para prevenir la infección (6).

Por último, frente al argumento de inadecuadas metodologías en los talleres sobre malaria, según Gramiccia, una razón para las limitaciones en la educación para el control de la malaria son los métodos empleados, generalmente métodos hablados que no son adecuadamente adaptados a las situaciones locales (13).

En conclusión aunque se reconozca la etiología, los síntomas, las causas, la población de alto riesgo para contraer la enfermedad, los lugares en donde se está expuesto y los periodos del año de mayor incidencia; esta información no es suficiente para que las personas actúen para prevenir y controlar la malaria, se deberán adelantar mas estudios que permitan entender como los factores comportamentales de las poblaciones expuesta a la malaria, pueden facilitar u obstaculizar las intervenciones de control en el Departamento del Amazonas Colombiano • 


\section{REFERENCIAS}

1. Heggenhougen K, Hackethal V, Vivek P, Espielman A. The Behavioural and social aspects of malaria and its control. UNDP/WorldBank/WHO Special Programme for Research \& Training Tropical Diseases (TDR). 2003.

2. Tanner M, Vlassoff C. Treatment-seeking behaviour for malaria: a typology based on endemicity and gender. Social Science \& Medicine.1998; 46:523-532

3. Bonilla E, Kuratomi L, Rodríguez P, Rodríguez A. Aspectos socioeconómicos de la malaria en Colombia. Salud y Desarrollo. Universidad de los Andes. Facultad de economía. Centro de Estudios Sobre Desarrollo Económico. CEDE; 1991.

4.Vieco J, Franky C, Echeverri J. Territorialidad Indígena y Ordenamiento en la Amazonía. Universidad Nacional de Colombia. Instituto Amazónico de Investigaciones; 2000.

5.WHO.TDR.Qualitative Research Methods. Resource papers for social and economic research in tropical diseases No.3; 1994.

6.Klein R, Wellwe S, Zeissig R, Knowledge, beliefs, and practices in relation to malaria transmission and.vector control in Guatemala. American Journal of Tropical Medicine and Hygiene 1995; 52:383-388.

7.Booth C, MacLean D, Knowledge, treatment-Seeking, and socioeconomic Impact of Malaria on the Essequibo coast of Guyana .MJM 20001; 6:17-25

8.Lipowsky R, kroeger A, Vazquez M. Sociomedical aspects of malaria control in Columbia. Social Science and Medicine.1992; 34:625-637

9. Rodriguez A, Penilla R, Rodriguez M, Hemingway L, Betanzos A, Hernandez J. Knowledge and beliefs about malaria transmission and practices for vector control in southern Mexico. Salud Pública Mex. 2003; 45(2):110-116.

10. McCombie SC. Treatment seeking for malaria a review of recent research. Social Science and Medicine. 1996; 43: 933-945.

11. Foster S. Pricing, distribution, and use of antimalarial drugs. Bulletin of the WHO. 1991; 69: 349-363.

12. Adera TD. Beliefs and traditional treatment of malaria in Kishe settiement area, southwest Ethiopia. EHP Library Malaria Bulletin 60:may 20-june 1;2003.

13. Gramiccia G. Health education in malaria control Why has it failed? World Health Forum. 1981; 2.385-393. 